TRANSACTIONS OF THE

AMERICAN MATHEMATICAL SOCIETY

Volume 357, Number 4, Pages 1341-1348

S 0002-9947(04)03493-2

Article electronically published on May 10, 2004

\title{
ON ADIC GENUS AND LAMBDA-RINGS
}

\author{
DONALD YAU
}

\begin{abstract}
Sufficient conditions on a space are given which guarantee that the $K$-theory ring is an invariant of the adic genus. An immediate consequence of this result about adic genus is that for any positive integer $n$, the power series ring $\mathbf{Z}\left[\left[x_{1}, \ldots, x_{n}\right]\right]$ admits uncountably many pairwise non-isomorphic $\lambda$-ring structures.
\end{abstract}

\section{IntRoduction AND STATEMENT OF RESUltS}

In this paper we study a problem about the adic genus of a space. For a nilpotent finite type space $X$, we denote by $\operatorname{Genus}(X)$, called the adic genus of $X$, the set of homotopy types of nilpotent finite type spaces $Y$ such that the $p$-completions of $X$ and $Y$ are homotopy equivalent for each prime $p$ and also their rationalizations are homotopy equivalent.

The adic genus of an infinite-dimensional space $X$ is often a very big set. For instance, Møller [11] proved that whenever $G$ is a compact connected non-abelian Lie group, the adic genus of its classifying space $B G$ is uncountably large. It is an important problem to find computable homotopy invariants which can distinguish between spaces with the same adic genus. Such a result was achieved by Notbohm and Smith [13. (see also 7]). Recall that a space $X$ is said to be an adic fake Lie group of type $G$ if (1) $X=\Omega B X$ is a finite loop space and (2) $B X$ lies in the adic genus of $B G$. Notbohm and Smith showed that if $X$ is an adic fake Lie group of type $G$, where $G$ is a simply-connected compact Lie group, then $B X$ is homotopy equivalent to $B G$ if and only if $K(B X) \cong K(B G)$ as $\lambda$-rings. (Here $K(Y)$ denotes the complex $K$-theory of $Y$.) In fact, Notbohm 12 showed that $K$-theory $\lambda$-rings classify the genus of $B G$, provided $G$ is a simply-connected compact Lie group. Note that what we call a $\lambda$-ring is what used to be called a "special" $\lambda$-ring.

With this result in mind, a natural question is then the following:

Is it really necessary to take into account the $\lambda$-operations in order to distinguish spaces in the adic genus of $B G$ ?

To describe our answer to this question, we need the following definition. A filtered ring is a commutative ring $R$ with unit together with a decreasing filtration

$$
R=I^{0} \supset I^{1} \supset I^{2} \supset \cdots
$$

of ideals such that $I^{m} I^{n} \subset I^{m+n}$. A map of filtered rings is required to respect filtrations. The filtration in the $K$-theory of a space $X$ is given by a skeletal

Received by the editors May 1, 2002 and, in revised form, August 1, 2003.

2000 Mathematics Subject Classification. Primary 55P15; Secondary 55N15, 55P60, 55S25.

Key words and phrases. Adic genus, lambda-rings. 
filtration of $X$; that is, $K(X)$ is filtered by the kernels

$$
I^{n}(X)=\operatorname{ker}\left(K(X) \rightarrow K\left(X_{n-1}\right)\right)
$$

of the restriction maps, where $X_{n-1}$ denotes the $(n-1)$-skeleton of $X$. By using the Cellular Approximation Theorem, it is easy to see that the filtered ring isomorphism type of a given space $X$ is well defined, although there are many different filtrations on $X$. Clearly, a map between two spaces induces a filtered ring map between the respective $K$-theories.

Our main result is then the following, which shows that for a torsion free classifying space, $K$-theory filtered ring cannot tell the difference between spaces in its adic genus. Consequently, $\lambda$-operations are necessary in order to distinguish these spaces.

Theorem 1.1. Let $X$ be a simply-connected space of finite type whose integral homology is torsion free and is concentrated in even dimensions and whose $K$-theory filtered ring is a finitely generated power series ring over $\mathbf{Z}$. If a space $Y$ belongs to the adic genus of $X$, then there exists a filtered ring isomorphism $K(X) \cong K(Y)$.

For example, in Theorem 1.1 the space $X$ can be $B S p(n)(n \geq 1), B S U(n)$ $(n \geq 2)$, or any finite product of copies of these spaces and of infinite complex projective space.

Remark 1.2. The proof of Theorem 1.1 is independent of Notbohm's result mentioned above. It consists of first showing the weaker statement that the filtered rings $K(Y) / I^{n}(Y)$ and $K(X) / I^{n}(X)$ are isomorphic for all $n$ sufficiently large. Since $K(Y)$ can be recovered from the quotients $K(Y) / I^{n}(Y)$ by taking inverse limits, a filtered ring analog of a classification result of Wilkerson [19] implies that to prove Theorem 1.1 it is sufficient to prove the triviality of a $\lim ^{1}$ set.

Remark 1.3. This theorem has a variant in which complex $K$-theory (resp. Z) is replaced with $K O^{*}$-theory (resp. $K O^{*}=K O^{*}(\mathrm{pt})$ ), provided the integral homology of $X$ (which is simply-connected of finite type) is torsion free and is concentrated in dimensions divisible by 4 (e.g. $X=B S p(n)$ ). This variant admits a proof which is essentially identical with that of Theorem 1.1 .

We now describe a purely algebraic application of Theorem 1.1 to the study of $\lambda$-ring structures over a fixed underlying ring. In fact, at least for algebraists, this algebraic result may be of even greater interest than the theorem above.

Now since the adic genus of $B U(n), n$ any positive integer, is uncountable [11, Theorems 1.1 and Notbohm's result alluded to above imply the following purely algebraic statement about $\lambda$-rings.

Corollary 1.4. Let $n$ be any positive integer. There exist uncountably many distinct isomorphism classes of $\lambda$-ring structures over the power series ring $\mathbf{Z}\left[\left[x_{1}, \ldots, x_{n}\right]\right]$ on $n$ indeterminates.

A few remarks are in order.

Remark 1.5. Notice that the way we obtain Corollary 1.4 is by combining three topological results about spaces in the genus of classifying spaces and their $\mathrm{K}$ theories, even though the statement of the result is purely algebraic. It would be nice if this statement can be demonstrated in an algebraic way without using spaces. 
Remark 1.6. As far as the author is aware, this result about $\lambda$-ring structures is new for every $n$. It exhibits a huge diversity of $\lambda$-ring structures over finitely generated power series rings. The author does not know of any reference in the literature that mentions the question of describing the $\lambda$-ring structures on power series rings.

Remark 1.7. The analogous question of how many $\lambda$-ring structures the polynomial ring $\mathbf{Z}[x]$ on one indeterminate supports has been studied by Clauwens [6]. Employing the theory of commuting polynomials, he showed that, in stark contrast to our corollary, there are essentially only two non-isomorphic $\lambda$-ring structures on the polynomial ring $\mathbf{Z}[x]$. We, however, have not been able to establish any connections between Clauwens' result and our Corollary 1.4. As pointed out by Clauwens, the same question for the polynomial ring on $n$ indeterminates, $n>1$, is still open.

This finishes the presentation of the results in this paper. The rest of the paper is organized as follows. In $\S 2$ we recall the definitions of a (filtered) $\lambda$-ring. The proof of Theorem 1.1 is given in 3 .

\section{2. $\lambda$-Rings AND Adams operations}

In this section, we recall the definitions of a (filtered) $\lambda$-ring. The reader is referred to Atiyah and Tall [3] or Knutson [9] for more information about $\lambda$-rings.

2.1. $\lambda$-rings. A $\lambda$-ring is a commutative ring $R$ with unit together with functions

$$
\lambda^{i}: R \rightarrow R
$$

for $i=0,1, \ldots$ satisfying the following properties: For any elements $r$ and $s$ in $R$ one has

$$
\begin{aligned}
& \text { - } \lambda^{0}(r)=1, \\
& \text { - } \lambda^{1}(r)=r, \lambda^{n}(1)=0 \text { for all } n>1, \\
& \text { - } \lambda^{n}(r+s)=\sum_{i=0}^{n} \lambda^{i}(r) \lambda^{n-i}(s), \\
& \text { - } \lambda^{n}(r s)=P_{n}\left(\lambda^{1}(r), \ldots, \lambda^{n}(r) ; \lambda^{1}(s), \ldots, \lambda^{n}(s)\right), \\
& \text { - } \lambda^{n}\left(\lambda^{m}(r)\right)=P_{n, m}\left(\lambda^{1}(r), \ldots, \lambda^{n m}(r)\right) .
\end{aligned}
$$

Here the $P_{n}$ and $P_{n, m}$ are certain universal polynomials with integer coefficients. Note that in the literature (for example, Atiyah and Tall [3]) the terminology "special" $\lambda$-ring is used.

A $\lambda$-ring map $f: R \rightarrow S$ between two $\lambda$-rings is a ring map between the underlying rings which respects the operations $\lambda^{i}: f \lambda^{i}=\lambda^{i} f(i \geq 0)$.

2.2. Filtered $\lambda$-rings. A filtered $\lambda$-ring is a filtered $\operatorname{ring} R=\left(R,\left\{I^{n}\right\}\right)$ which is also a $\lambda$-ring such that the ideals $I^{n}$ are all closed under the operations $\lambda^{i}(i>0)$.

A filtered $\lambda$-ring map is a $\lambda$-ring map which is also a filtered ring map.

\section{Proof of Theorem 1.1}

In this section we prove Theorem 1.1 which consists of a few lemmas. Throughout this section we work in the category of filtered rings.

We will make use of the following observation, whose proof is a straightforward adaptation of Wilkerson's proof of the classification theorem [19] Theorem I] of spaces of the same $n$-type for all $n$. Now let $X$ be as in Theorem 1.1. 
Lemma 3.1 (Wilkerson). There is a bijection between the following two pointed sets:

(1) The pointed set of isomorphism classes of filtered rings $\left(R,\left\{I_{n}\right\}\right)$ with the following properties:

(a) The natural map $R \rightarrow \lim _{n} R / I_{n}$ is an isomorphism, and

(b) $R / I_{n}$ and $K(X) / K_{n}(X)$ are isomorphic as filtered rings for all $n>0$.

(2) The pointed set $\lim _{n}^{1} \operatorname{Aut}\left(K(X) / K_{n}(X)\right)$.

Here Aut(-) denotes the group of filtered ring automorphisms, and the $\varliminf^{1}$ of a tower of not necessarily abelian groups is as defined in Bousfield-Kan [5].

The two lemmas below will show that, for every space $Y$ in the genus of $X$, the object $K(Y)$ lies in the first pointed set in Lemma 3.1 .

Lemma 3.2. For every space $Y$ in the genus of $X$ (as in Theorem 1.1), the natural map

$$
K(Y) \rightarrow \lim _{j} K(Y) / K_{j}(Y)
$$

is an isomorphism.

Proof. According to [4, 2.5 and 7.1] the natural map from $K(Y)$ to $\varliminf_{j} K(Y) / K_{j}(Y)$ is an isomorphism if the following condition holds:

$$
\varliminf_{r}^{1} E_{r}^{p, q}=0 \text { for all pairs }(p, q) \text {. }
$$

Here $E_{r}^{*, *}$ is the $E_{r}$-term in the $K^{*}$-Atiyah-Hirzebruch spectral sequence (AHSS) for $Y$. This condition is satisfied, in particular, when the AHSS degenerates at the $E_{2}$-term. Thus, to prove (3.1) it suffices to show that $H^{*}(Y ; \mathbf{Z})$ is concentrated in even dimensions, since in that case there is no room for differentials in the AHSS. So pick an odd integer $N$. We must show that

$$
H^{N}(Y ; \mathbf{Z}) \cong 0 \text {. }
$$

This last condition holds because, as $Y$ lies in the genus of $X$, the integral cohomology groups of the two spaces are isomorphic.

This finishes the proof of Lemma 3.2

Lemma 3.3. For every space $Y$ in the genus of $X$ (as in Theorem 1.1), the filtered rings $K(Y) / K_{n}(Y)$ and $K(X) / K_{n}(X)$ are isomorphic for each $n>0$.

Proof. We have to show that for each $j>0$ there is an isomorphism of filtered rings

$$
K(Y) / K_{j}(Y) \cong K(X) / K_{j}(X) .
$$

It follows from the hypothesis that for each $j>0$, the filtered ring $K(Y) / K_{j}(Y)$ belongs to $\operatorname{Genus}\left(K(X) / K_{j}(X)\right)$, where $\operatorname{Genus}(R)$ for a filtered ring $R$ is defined in terms of $R \otimes \mathbf{Q}$ and $R \otimes \widehat{\mathbf{Z}}$ in exactly the same way the genus of a space is defined. To finish the proof we will adapt two results of Wilkerson [20, 3.7 and 3.8], which we now recall.

For a nilpotent finite type space $X$, Wilkerson showed that there is a surjection

$$
\sigma: \operatorname{Caut}\left(\hat{X}_{0}\right) \rightarrow \operatorname{Genus}(X)
$$

where $\hat{X}_{0}$ is the rationalization of the formal completion $\hat{X}=\prod_{p} X_{p}^{\wedge}$ of $X$. Notice that each homotopy group $\pi_{*}\left(\hat{X}_{0}\right)$ is a $\mathbf{Q} \otimes \widehat{\mathbf{Z}}$-module, and $\operatorname{Caut}\left(\hat{X}_{0}\right)$ is by definition 
the group of homotopy classes of self-homotopy equivalences of $\hat{X}_{0}$ whose induced maps on homotopy groups are $\mathbf{Q} \otimes \widehat{\mathbf{Z}}$-module maps.

Note that the definitions Genus(-) and Caut(-) also make sense in both the categories of nilpotent groups and of filtered rings. For instance, if $R=\left(R,\left\{I^{n}\right\}\right)$ is a filtered ring, then $\operatorname{Caut}(R \otimes \mathbf{Q} \otimes \widehat{\mathbf{Z}})$ is the group of filtered ring automorphisms of $R \otimes \mathbf{Q} \otimes \widehat{\mathbf{Z}}$ which are also $\mathbf{Q} \otimes \widehat{\mathbf{Z}}$-module maps. Now if $A$ is a finitely generated abelian group, then Wilkerson showed that for any class $[\varphi] \in \operatorname{Caut}(A \otimes \mathbf{Q} \otimes \widehat{\mathbf{Z}})$, the image $\sigma([\varphi])$ is isomorphic to $A$ as groups; that is, the image of $\sigma$ is constant at $A$. The main point here is that the double coset

$$
\operatorname{Aut}(A \otimes \widehat{\mathbf{Z}}) \backslash \operatorname{Caut}(A \otimes \mathbf{Q} \otimes \widehat{\mathbf{Z}}) / \operatorname{Aut}(A \otimes \mathbf{Q})
$$

is trivial because every element in the middle term can be written as a product of two elements, one from each of the left and the right terms.

It is straightforward to adapt Wilkerson's proofs of these results to show that for each $j$ the map

$$
\sigma: \operatorname{Caut}\left(\left(K(X) / K_{j}(X)\right) \otimes \mathbf{Q} \otimes \widehat{\mathbf{Z}}\right) \rightarrow \operatorname{Genus}\left(K(X) / K_{j}(X)\right)
$$

is surjective and that the image of $\sigma$ is constant at $K(X) / K_{j}(X)$. In other words, the genus of $K(X) / K_{j}(X)$ is the one-point set. Therefore, $K(Y) / K_{j}(Y)$ is isomorphic to $K(X) / K_{j}(X)$.

This finishes the proof of Lemma 3.3 .

In view of Lemma 3.1, to finish the proof of Theorem 1.1 we are only left to show that the classifying object

$$
\varliminf_{n}^{1} \operatorname{Aut}\left(K(X) / K_{n}(X)\right)
$$

is the one-point set. To do this, it suffices to show that almost all of the structure maps in the tower are surjective. This is shown in the following lemma.

Lemma 3.4. The maps

$$
\operatorname{Aut}\left(K(X) / K_{j+1}(X)\right) \rightarrow \operatorname{Aut}\left(K(X) / K_{j}(X)\right)
$$

are surjective for all $j$ sufficiently large.

Proof. First note that by hypothesis the $K$-theory filtered ring of $X$ has the form

$$
K(X)=\mathbf{Z}\left[\left[c_{1}, \ldots, c_{n}\right]\right]
$$

in which the generators $c_{i}$ are algebraically independent over $\mathbf{Z}$ and $K_{j}(X)$ is the ideal generated by the monomials of filtrations at least $j$. Suppose that $d_{i}$ is the largest integer $k$ for which $c_{i}$ lies in filtration $k$. Let $N$ be the integer

$$
N=\max \left\{d_{i}: 1 \leq i \leq n\right\}+1 \text {. }
$$

We will show that the structure maps

$$
\operatorname{Aut}\left(K(X) / K_{j+1}(X)\right) \rightarrow \operatorname{Aut}\left(K(X) / K_{j}(X)\right)
$$

are surjective for all $j>N$.

So fix an integer $j>N$ and pick a filtered ring automorphism $\sigma$ of $K(X) / K_{j}(X)$. We must show that $\sigma$ can be lifted to a filtered ring automorphism of $K(X) /$ $K_{j+1}(X)$. For $1 \leq i \leq n$ pick any lift of the element $\sigma\left(c_{i}\right)$ to $K(X) / K_{j+1}(X)$ and 
call it $\hat{\sigma}\left(c_{i}\right)$. Since the various quotient filtered rings $K(X) / K_{s}(X)$ are still generated by (the images of) the $c_{i}$, we may and will require that $\hat{\sigma}\left(c_{i}\right)$ have filtration exactly $d_{i}$. Now since the elements $c_{i}$ generate $K(X) / K_{j+1}(X)$, one can extend $\hat{\sigma}$ to a well-defined filtered ring endomorphism. It will be a desired lift of $\sigma$ once it is shown to be bijective.

To show that

$$
\hat{\sigma}: K(X) / K_{j+1}(X) \rightarrow K(X) / K_{j+1}(X)
$$

is surjective, it suffices to show that the image of each $c_{i}$ in $K(X) / K_{j+1}(X)$ lies in the image of $\hat{\sigma}$, since $K(X) / K_{j+1}(X)$ is generated as a filtered ring by the images of the $c_{i}$. So fix an integer $i$ with $1 \leq i \leq n$. We know that there exists an element $g_{i} \in K(X) / K_{j}(X)$ such that

$$
\sigma\left(g_{i}\right)=c_{i} .
$$

Pick any lift of $g_{i}$ to $K(X) / K_{j+1}(X)$, call it $g_{i}$ again, and observe that (3.5) implies that

$$
\hat{\sigma}\left(g_{i}\right)=c_{i}+\alpha_{i}
$$

in $K(X) / K_{j+1}(X)$ for some element $\alpha_{i} \in K_{j}(X) / K_{j+1}(X)$. We will alter $g_{i}$ to obtain a $\hat{\sigma}$-pre-image of $c_{i}$ as follows.

Observe that the ideal $K_{j}(X) / K_{j+1}(X)$ is generated by certain monomials in $c_{1}, \ldots, c_{n}$. Namely, the monomials

$$
\mathbf{c}^{\mathbf{i}}=c_{1}^{i_{1}} \cdots c_{n}^{i_{n}}, \quad \mathbf{i}=\left(i_{1}, \ldots, i_{n}\right) \in J_{j},
$$

where $J_{j}$ is the set of ordered $n$-tuples $\mathbf{i}=\left(i_{1}, \ldots, i_{n}\right)$ of nonnegative integers satisfying

$$
\mathbf{d} \cdot \mathbf{i}=\sum_{l=1}^{n} d_{l} i_{l}=j .
$$

Thus, for every element $\mathbf{i}$ in the set $J_{j}$, there exists a corresponding integer $a_{\mathbf{i}}$ such that we can write the element $\alpha_{i}$ as the sum

$$
\alpha_{i}=\sum_{\mathbf{i} \in J_{j}} a_{\mathbf{i}} \mathbf{c}^{\mathbf{i}} .
$$

Now define the element $\bar{g}_{i}$ in $K(X) / K_{j+1}(X)$ by the formula

$$
\bar{g}_{i} \stackrel{\text { def }}{=} g_{i}-\sum_{\mathbf{i} \in J_{j}} a_{\mathbf{i}} \mathbf{g}^{\mathbf{i}}, \quad \text { where } \quad \mathbf{g}^{\mathbf{i}}=g_{1}^{i_{1}} \cdots g_{n}^{i_{n}} .
$$

We claim that $\bar{g}_{i}$ is a $\hat{\sigma}$-pre-image of $c_{i}$. That is, we claim that

$$
\hat{\sigma}\left(\bar{g}_{i}\right)=c_{i} \text { in } K(X) / K_{j+1}(X) .
$$

In view of (3.6), (3.8), and (3.9), it clearly suffices to prove the following equality for each element $\mathbf{i}$ in $J_{j}$ :

$$
\hat{\sigma}\left(\mathbf{g}^{\mathbf{i}}\right)=\mathbf{c}^{\mathbf{i}} \quad \text { in } \quad K(X) / K_{j+1}(X) .
$$


Now in the quotient $K(X) / K_{j+1}(X)$, one computes

$$
\begin{aligned}
\hat{\sigma}\left(\mathbf{g}^{\mathbf{i}}\right) & =\prod_{j=1}^{n} \hat{\sigma}\left(g_{j}\right)^{i_{j}} \\
& =\prod_{j=1}^{n}\left(c_{j}+\alpha_{j}\right)^{i_{j}} \quad \text { by (3.6) } \\
& =\mathbf{c}^{\mathbf{i}}+(\text { terms of filtrations }>j) \\
& =\mathbf{c}^{\mathbf{i}} .
\end{aligned}
$$

This proves (3.11), and hence (3.10), and therefore $\hat{\sigma}$ is surjective.

It remains to show that $\hat{\sigma}$ is injective. Since any surjective endomorphism of a finitely generated abelian group is also injective and since $K(X) / K_{j+1}(X)$ is a finitely generated abelian group, it follows that $\hat{\sigma}$ is injective as well. Thus, $\hat{\sigma}$ is a filtered-ring automorphism of $K(X) / K_{j+1}(X)$ and is a lift of $\sigma$.

This finishes the proof of Lemma 3.4

This proof of Theorem 1.1 is complete.

\section{ACKNOWLEDGEMENTS}

The results in this paper constitute part of the author's 2002 Ph.D. thesis at MIT. The author expresses his sincerest gratitude to his advisor, Professor Haynes Miller. The author also thanks the anonymous referee for his comments and suggestions.

\section{REFERENCES}

[1] J. F. Adams, Localisation and completion, University of Chicago Press, Chicago, Ill., 1975. MR 54:8621

[2] M. F. Atiyah, Power operations in K-theory, Quart. J. Math. Oxford 17 (1966) 163-193. MR 34:2004

[3] M. F. Atiyah and D. O. Tall, Group representations, $\lambda$-rings and the $J$-homomorphism, Topology 8 (1969) 253-297. MR 39:5702

[4] J. Michael Boardman, Conditionally convergent spectral sequences, Contemp. Math. 239, Amer. Math. Soc., Providence, RI, 1999, pp. 49-84. MR 2000m:55024

[5] A. K. Bousfield and D. M. Kan, Homotopy limits, completions and localizations, Lecture Notes in Mathematics, Vol. 304, Springer-Verlag, Berlin-New York, 1972. MR 51:1825

[6] F. J. B. J. Clauwens, Commuting polynomials and $\lambda$-ring structures on $\mathbf{Z}[x]$, J. Pure Appl. Algebra 95 (1994) 261-269. MR 95k:13008

[7] A. Jeanneret and A. Osse, The $K$-theory of $p$-compact groups, Comment. Math. Helv. 72 (1997) 556-581. MR 99d:55007

[8] D. C. Johnson and W. S. Wilson, $B P$ operations and Morava's extraordinary $K$-theories, Math. Z. 144 (1975) 55-75. MR 51:14025

[9] D. Knutson, $\lambda$-rings and the representation theory of the symmetric group, Lecture Notes in Mathematics, Vol. 308. Springer-Verlag, Berlin-New York, 1973. MR 51:679

[10] C. A. McGibbon, The Mislin genus of a space, The Hilton Symposium 1993 (Montreal, PQ), 75-102, CRM Proc. Lecture Notes, 6, Amer. Math. Soc., Providence, RI, 1994. MR 96a:55018

[11] J. M. Møller, The normalizer of the Weyl group, Math. Ann. 294 (1992) 59-80. MR 94b:55010

[12] D. Notbohm, Maps between classifying spaces and applications, J. Pure Appl. Algebra 89 (1993) 273-294. MR 95c:55019

[13] D. Notbohm and L. Smith, Fake Lie groups and maximal tori. I - III, Math. Ann. 288 (1990) 637-661, 663-673 and 290 (1991) 629-642.

[14] D. L. Rector, Loop structures on the homotopy type of $S^{3}$, Symposium on Algebraic Topology (Battelle Seattle Res. Center, Seattle, Wash., 1971), pp. 99-105. Lecture Notes in Math., Vol. 249, Springer, Berlin, 1971. MR 49:3916 
[15] Subgroups of finite dimensional topological groups, J. Pure Appl. Algebra 1 (1971) 253-273. MR 46:889

[16] Y. Shitanda, Uncountably many loop spaces of the same $n$-type for all $n$. I and II, Yokohama Math. J. 41 (1993) 17-24 and 41 (1994) 85-93.

[17] Uncountably many infinite loop spaces of the same $n$-type for all $n$, Math. J. Okayama Univ. 34 (1992) 217-223. MR 95j:55019

[18] S Spaces of the same clone type, J. Math. Soc. Japan 48 (1996) 705-714. MR 97i:55015

[19] C. Wilkerson, Classification of spaces of the same $n$-type for all $n$, Proc. Amer. Math. Soc. 60 (1976) 279-285. MR 57:13930

[20] _ Applications of minimal simplicial groups, Topology 15 (1976) 111-130. MR 53:6551

$[21]$, Lambda-rings, binomial domains, and vector bundles over $C P(\infty)$, Comm. Algebra 10 (1982) 311-328. MR 83f:55003

Department of Mathematics, University of Illinois at Urbana-Champaign, 1409

W. Green Street, Urbana, Illinois 61801

E-mail address: dyau@math.uiuc.edu 